\title{
Interactions between astronomical ephemerides and society
}

\author{
Jean-Eudes Arlot \\ IMCCE, Observatoire de Paris, CNRS, \\ 77 avenue Denfert-Rochereau, 75014 Paris, France \\ email: arlot@imcce.fr
}

\begin{abstract}
Ephemerides are regularly made by astronomers for their own uses. However, the general public is also interested, as well as official organisms, because of the interactions of ephemerides with society. Astronomers in charge of the making of calendars and keeping the time are of great importance every day. Their calculations are also required for the positioning of ships and airplanes. Some ephemerides are also requested by the general public.
\end{abstract}

Keywords. ephemerides, Sun, Moon, planets, GPS, navigation

\section{Introduction}

Astronomical ephemerides are proposed first to astronomers making observations and to the space agencies preparing or analysing space missions and are the results of the scientific research.

However, some information coming from ephemerides may have an influence on society. The general public is interested, and needs, some astronomical informations. What ephemerides are the most requested ones by the general public because of their implications, by whom and for what purpose? This last point is sufficiently important to deserve a law charging an official public laboratory to do this work and to answer the requests of society.

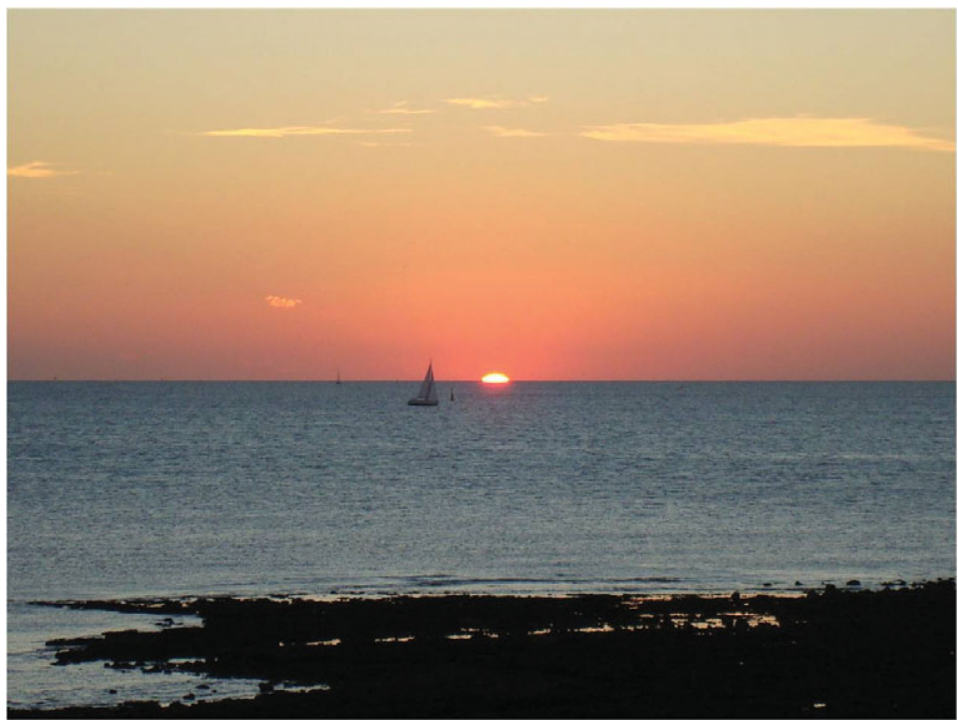

Figure 1. The time of sunset is the main request from the general public to astronomers. 


\section{What are the ephemerides?}

Ephemerides are predictions of the positions of the Sun, Moon, planets, moving objects in the sky and also stars. From these ephemerides, we predict phenomena such as sunrises, sunsets (Fig. 1), eclipses (Fig. 6), ... Ephemerides may have several forms such as tabulated values of positions in spherical coordinates on the celestial sphere needing interpolation for accurate positions for a given date, or as drawing of configurations of the aspect (Fig. 3) of bodies in the sky. For the general public, ephemerides are mainly the dates of sunrises and sunsets provided in calendars and almanacs available at the beginning of the year.

Almanacs and calendars are provided from centuries as soon as the time (days, years) should be counted. Ephemerides in the sense given above are published only since the 17th century and appeared first in Europe (Fig. 2). At Paris observatory, the first ephemerides were published starting in 1679 in the "Connaissance des temps" with no interruption to nowadays (Fig. 5). At first, positions of the Sun and the Moon were provided together with the major phenomena such as the eclipses of the Sun and the Moon. Precise predictions of the eclipses of the Sun were possible only from the 18th century. The dates of some phenomena such as eclipses of the satellites of Jupiter were regularly published since their observations were used as reference clock.

One of the most strategic uses of the ephemerides is for navigation at sea needing positions of stars and planets to know where the ships are. Specific nautical almanacs are designed for that purpose and the first astronomical observatories (Paris, Greenwich) were built mainly for that purpose. Nowadays, public organisms are in charge of the publication of ephemerides which may be different from the making of ephemerides now depending on high level scientific research. In the USA, the Naval Observatory is in charge of the official ephemerides and the Jet Propulsion Laboratory produces ephemerides

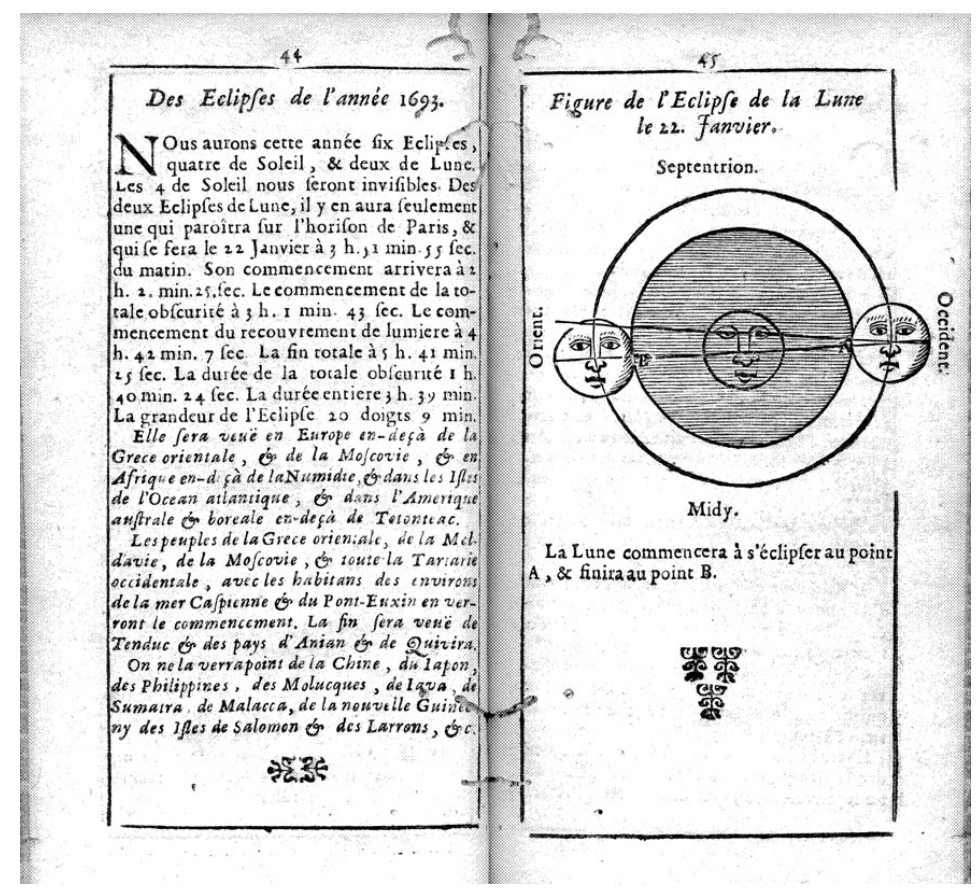

Figure 2. Ephemerides are published since the 17th century (here the Connaissance des temps published by Paris Observatory since 1679). 
for space purposes. In Russia, the Institute for Applied Astronomy in Saint Petersburg is building planetary ephemerides. In France, the Institut de Mécanique Céleste et de Calcul des Ephémerides (under the auspices of the Bureau des Longitudes created by the French revolution) belonging now to Paris Observatory is in charge of the official national ephemerides and also of the making of ephemerides for space purpose. Other official ephemerides bureaus depending on astronomers are in Oxford (Great Britain), Nanjing (Purple Mountain Observatory in China), Tokyo (Hydrographic Department in Japan) . If many countries have their own ephemerides bureaus, most of them are asking other bureaus to provide them the data and have not enough astronomers to make the ephemerides.

\section{The different forms of ephemerides}

\subsection{Printed ephemerides}

Printed ephemerides are tabulated ephemerides needing interpolations. They are used when no accurate positions are requested or for checking calculations made thanks to softwares on computers.

In France, our printed official ephemerides (Fig. 5) are:

- Connaissance des temps, providing the best data for the main objects of the Solar system

- Annuaire du Bureau des longitudes, providing data for the general public and for the other almanacs needing astronomical data

- Ephémerides nautiques for nautical use at sea. They are adapted for positioning, needing as less as possible calculations.

\subsection{Electronic ephemerides}

Near the printed ephemerides are the ephemerides provided by softwares on computers or through Internet. They are more and more used by the users of ephemerides. A web server may provide many different types of ephemerides, positions, configurations (Fig. 3),

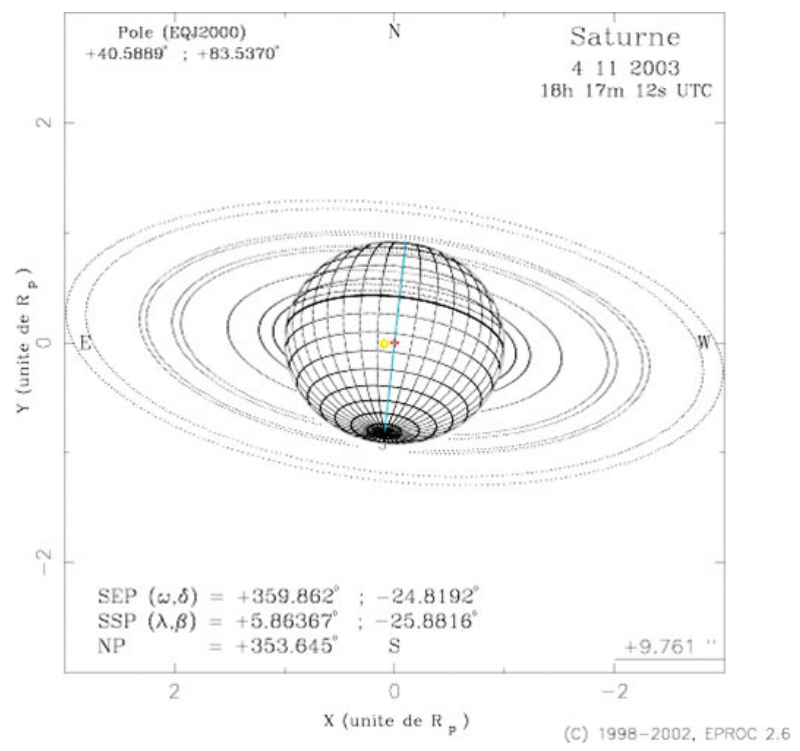

Figure 3. Ephemerides may be under the form of configurations. 
phenomena, for all solar system objects that is difficult in a printed ephemerides. The Web servers of the major laboratories are updated very often thanks to the results of the scientific research.

The ephemerides of the IMCCE are available at www.imcce.fr

\section{The users of ephemerides}

\section{- Astronomers}

Astronomers are the first users of ephemerides: they need to know precisely the positions of the objects on the celestial sphere in order to be able to observe with their telescopes and to find easily and to follow their objects. Optical telescopes, radio telescopes (Fig. 4) and telescopes in orbit around the Earth need continuously ephemerides.

- Space agencies

When planning a space mission in the solar system, the space agencies need to know exactly the positions of all the solar system bodies in order to optimize the navigation of the space probes. After the mission, ephemerides are still needed in order to understand and to use the data provided by the space probes.

- Ships and airplanes for positioning

In spite of the GPS system, it may be necessary to have an independent system of positioning. The stars, the planets, the Sun, the Moon are available for everybody and allow this purpose, providing that the astronomers calculate the positions of these objects allowing sailors to calculate their position. Note that the civilian air planes no more use astronomical ephemerides for positioning. Only the military air force uses it.

- The civilian society

The main demand of the general public is calendars, sunrises and sunsets (Fig. 1), then eclipses (Fig. 6), phases of the Moon, dates of the seasons and after that, positions of astronomical bodies. The calendar is made from astronomical data: some holidays such
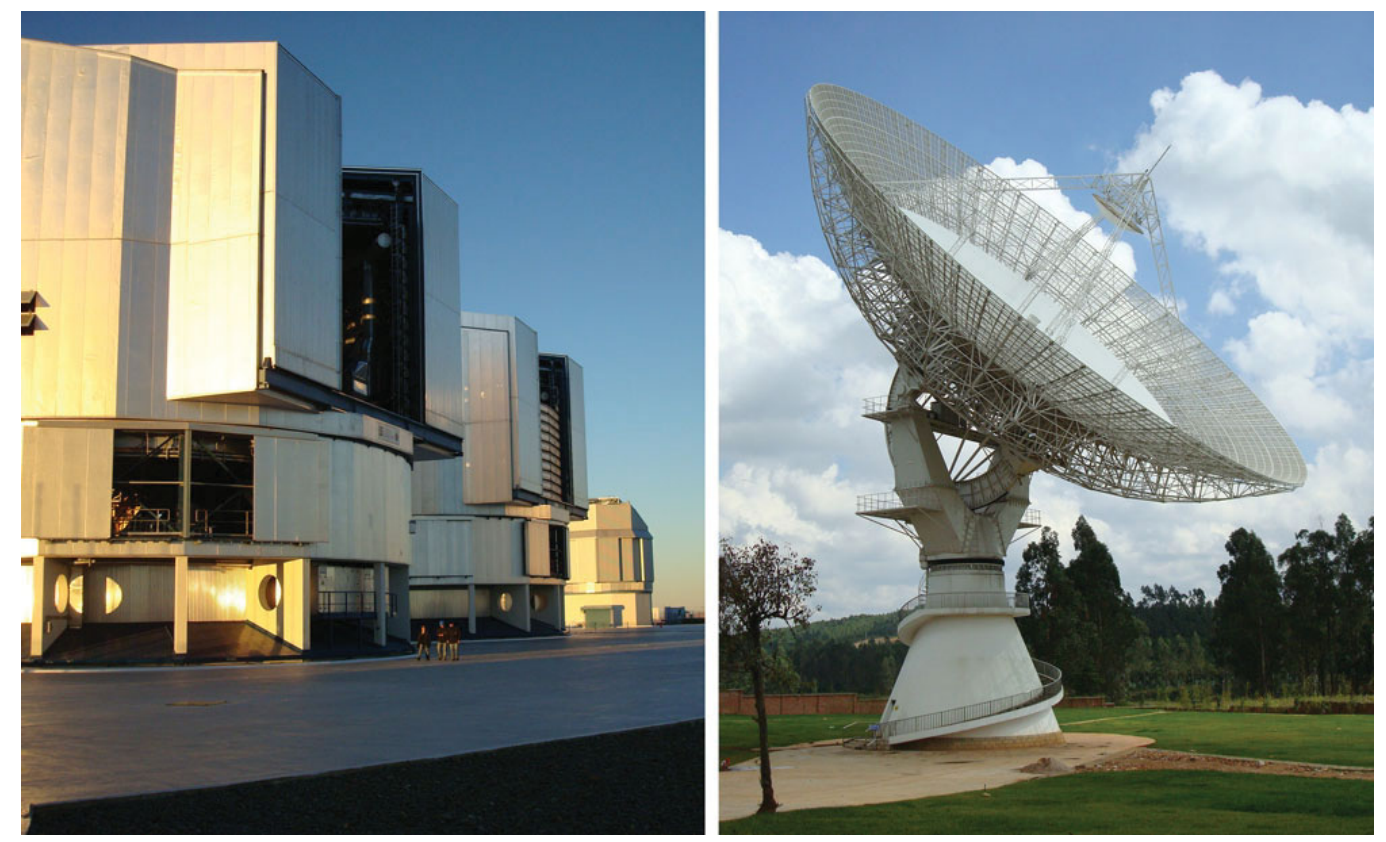

Figure 4. Optical and radio telescopes need ephemerides for accurate guiding. 
as Easter, depend on a theoretical Moon. The time scale that we use everyday is also deduced from the ephemerides of the Earth in its diurnal rotation.

Insurance companies and lawyers may ask astronomers for expertise: from a photography, the astronomers may use the shadows to date them. After an accident, the Sun may be responsible of some accident due to blooming and the astronomers may calculate the exact position of the Sun and its azimuth and elevation at the time of the accident. Movie makers need to know the exact position of the Sun in order to be able to use the light when taking pictures. Architects use to design their buildings with a specific orientation towards the Sun, especially when using the Sun for heating water or for the making of electricity. Private people have some curiosity concerning the sky they look at: they ask astronomers to know what is this bright star in the sky or at what time will be the sunrise because hunting and fishing are allowed only after the sunrise which is not always visible. Historians and archaeologists ask the astronomers to know the configurations of the Sun, the Moon or the stars near the old buildings and ancient graves which were supposed to be oriented in a specific direction. Sunrises and sunsets depend on the location of the observer and calculations are necessary for each specific site. Each airport needs also these calculations for each day of the year. At least, amateur astronomers are asking also for information allowing them to make interesting observations.

Statistics on the keywords on the Internet server of IMCCE are as follows: during one month, 'calendar' is requested 5600 times, 'Sun' 2900 times, 'Moon' 2300 times, 'eclipses' 1800 times and 'seasons' 1630 times.
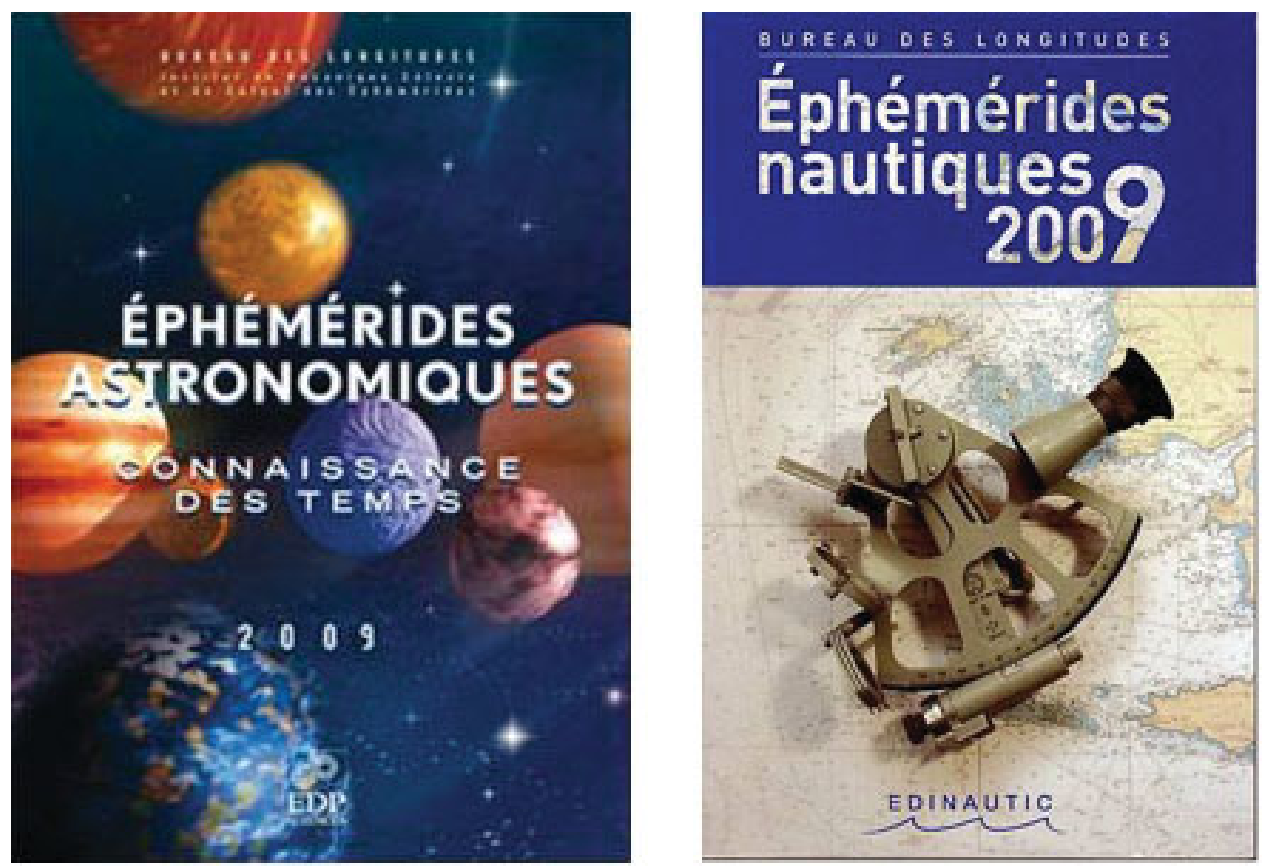

Figure 5. The Connaissance des temps and the French nautical almanac contain ephemerides for positioning at sea. 


\section{Conclusion: the relationship between astronomer and the general public}

The general public is very confident in the calculations and predictions made by the astronomers. Most of time they did not understand that astronomers make calculations with some uncertainties. The accuracy of the ephemerides is not understood by the general public. The occurrence of the eclipses at very precise dates accurately predicted by the astronomers encourages the general public to see ephemerides as the truth. Then, we encourage astronomers to publish information explaining their work to the general public, especially the making of ephemerides which gathers theoreticians and observers: the high level of accuracy of astronomical predicted phenomena and positions is the result of very long research work as explained in the volume edited by Simon et al. (1997).

\section{References}

Simon, J.L., Chapront-Touzé, M., Morando, B., \& Thuillot, W. (eds.) 1997, Introduction aux ephemerides astronomiques (Les Ulis: EDP Sciences)

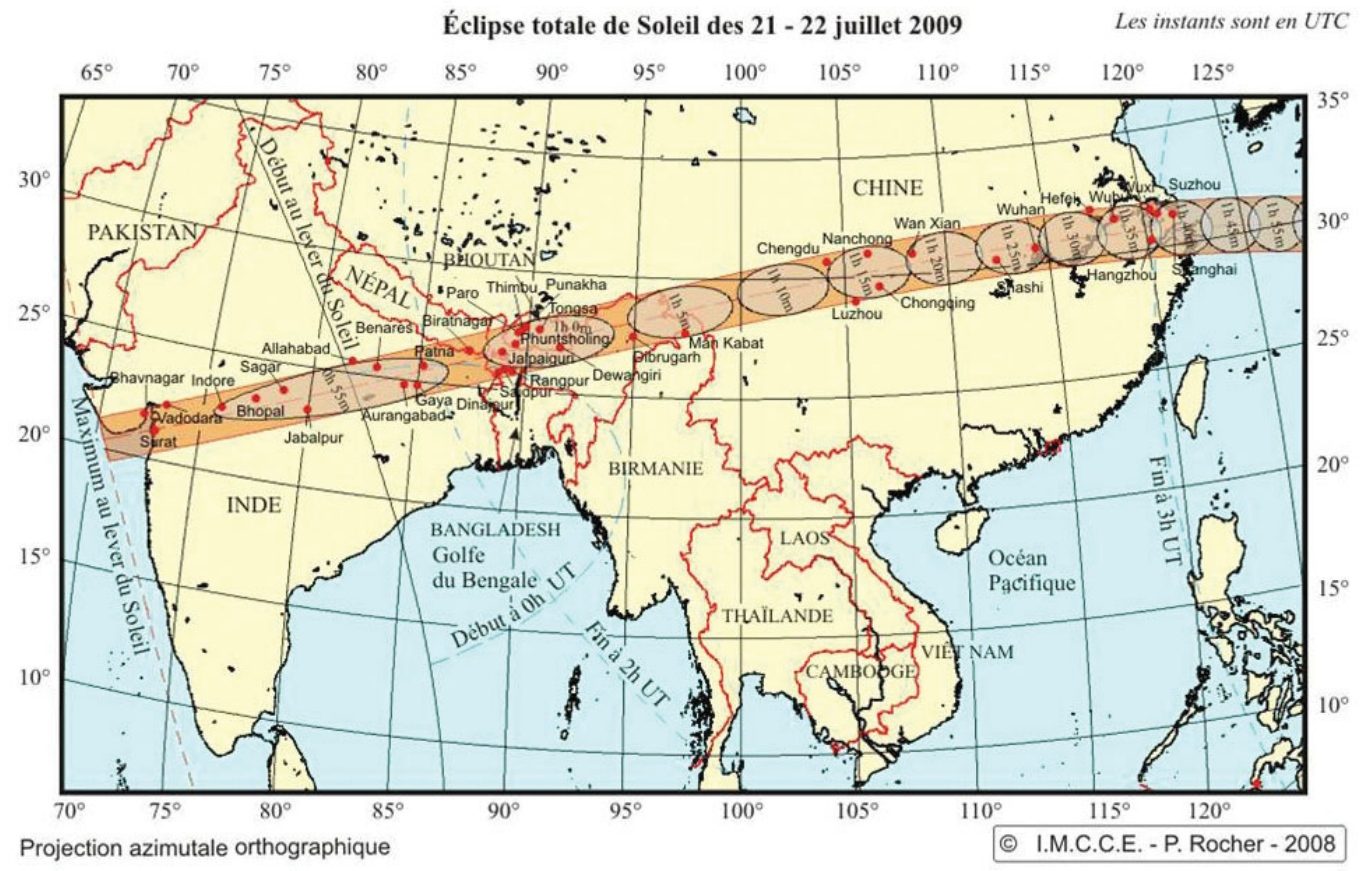

Figure 6. The predictions of eclipses provide the path of the lunar shadow on Earth. 